\title{
Social Jetlag Changes During the COVID-19 Pandemic as a Predictor of Insomnia - A Multi- National Survey Study
}

Luiz Eduardo Mateus Brandão, Teemu Martikainen, (iD) Illona Merikanto, (iD) ${ }^{2-4}$ Brigitte Holzinger, (iD ${ }^{5,6}$ Charles M Morin, iD ${ }^{7-9}$ Colin A Espie, (iD) ${ }^{10,11}$ Courtney J Bolstad, (iD) 12 Damien Leger, (iD) ${ }^{13}$ Frances Chung, (iD) ${ }^{14}$ Giuseppe Plazzi, iD ${ }^{15}$ Yves Dauvilliers, (iD) ${ }^{16}$ Kentaro Matsui, (D) 17,18 Luigi De Gennaro, iD ${ }^{19-21}$ Mariusz Sieminski, (iD) ${ }^{22}$ Michael R Nadorff, ${ }^{12,23}$ Ngan Yin Chan, iD ${ }^{24}$ Yun Kwok Wing, (iD ${ }^{24}$ Sérgio Arthuro MotaRolim, (iD) ${ }^{25-27}$ Yuichi Inoue, (iD) ${ }^{28}$ Markku Partinen, (D) ${ }^{29,30}$ Christian Benedict, (iD) ${ }^{31}$ Bjorn Bjorvatn, iD ${ }^{32,33}$ Jonathan Cedernaes (iD) ${ }^{1,34}$

'Department of Medical Sciences, Uppsala University, Uppsala, Sweden; ${ }^{2}$ Department of Public Health Solutions, Finnish Institute for Health and Welfare, Helsinki, Finland; ${ }^{3}$ Department of Psychology and Logopedics, University of Helsinki, Helsinki, Finland; ${ }^{4}$ Orton Orthopaedic Hospital, Helsinki, Finland; ${ }^{5}$ ZKSchlafcoaching, Medical University Vienna,

Vienna, Austria; ${ }^{6}$ Institute for Consciousness and Dream Research, Vienna, Austria; ${ }^{7}$ École de Psychologie, Université Laval, Québec,

Canada; ${ }^{8}$ Centre d'étude des troubles du sommeil, Université Laval, Québec, Canada;

${ }^{9}$ Centre de recherche CERVO/Brain Research

Center, Université Laval, Québec, Canada;

${ }^{10}$ Sleep \& Circadian Institute, University of

Oxford, Oxford, UK; " 'Nuffield Department of

Clinical Neurosciences, University of Oxford,

Oxford, UK; ${ }^{12}$ Department of Psychology,

Mississippi State University, Starkville, MS, USA

${ }^{13}$ APHP, VIFASOM, Hôtel-Dieu, Centre du Sommeil et de la Vigilance, Université de Paris, Paris, France; ${ }^{14}$ Department of Anesthesia and Pain Medicine, Toronto Western Hospital, University Health Network, University of Toronto, Toronto, Ontario, Canada;

${ }^{15}$ Department of Biomedical, Metabolic and Neural Science, University of Modena and Reggio Emilia, Modena, Italy; ${ }^{16}$ Sleep-Wake Disorders Center, Department of Neurology, Gui-de-Chauliac Hospital, Institute for Neurosciences of Montpellier INM, INSERM, University of Montpellier, Montpellier, France;

${ }^{17}$ National Center of Neurology and Psychiatry National Institute of Mental Health,

Department of Clinical Laboratory and
Purpose: Lifestyle and work habits have been drastically altered by restrictions due to the COVID-19 pandemic. Whether the associated changes in sleep timing modulate the risk of suffering from symptoms of insomnia, the most prevalent sleep disorder, is however incompletely understood. Here, we evaluate the association between the early pandemic-associated change in 1) the magnitude of social jetlag (SJL) - ie, the difference between sleep timing on working vs free days - and 2) symptoms of insomnia.

Patients and Methods: A total of 14,968 anonymous participants (mean age: 40 years; $64 \%$ females) responded to a standardized internet-based survey distributed across 14 countries. Using logistic multivariate regression, we examined the association between the degree of social jetlag and symptoms of insomnia, controlling for important confounders like social restriction extension, country specific COVID-19 severity and psychological distress, for example.

Results: In response to the pandemic, participants reported later sleep timing, especially during workdays. Most participants (46\%) exhibited a reduction in their SJL, whereas $20 \%$ increased it; and $34 \%$ reported no change in SJL. Notably, we found that both increased and decreased SJL, as a result of the COVID-19 pandemic, were associated with later sleep midpoint (indicating a later chronotype) as well as more recurrent and moderate-to-severe symptoms of insomnia (about 23 $54 \%$ higher odds ratio than subjects with unchanged SJL). Primarily those with reduced SJL shifted their bedtimes to a later timepoint, compared with those without changes in SJL.

Conclusion: Our findings offer important insights into how self-reported changes to the stability of sleep/wake timing, as reflected by changes in SJL, can be a critical marker of the risk of experiencing insomnia-related symptoms - even when individuals manage to reduce their social jetlag. These findings emphasize the clinical importance of analyzing sleep-wake regularity.

Keywords: BNSQ, ISI, midsleep, MSFsc, PHQ-4, psychological distress

\section{Introduction}

Stay-at-home orders and broad societal changes have greatly altered the degree of day-to-day social interactions and work schedules during the COVID-19 pandemic. This has been demonstrated to alter sleep schedules as well as the degree of social jetlag (SJL, the mismatch in sleep midpoint between work and free days) at the population-level. ${ }^{1-7}$ How this is related to sleep disorders, such as insomnia, has however remained largely unexplored.

Leone et $\mathrm{al}^{4}$ found that age and work status modulate the extent to which bedtime and wake-up time were altered during the early part of the COVID-19 
Department of Sleep-Wake Disorders, Tokyo, Japan; ${ }^{18}$ Tokyo Women's Medical University, Department of Psychiatry, Tokyo, Japan; ${ }^{19}$ Department of Psychology, Sapienza University of Rome, Rome, Italy; ${ }^{20}$ IRCCS Fondazione Santa Lucia, Rome, Italy; ${ }^{21}$ IRCCS Istituto delle Scienze Neurologiche di Bologna, Bologna, Italy; ${ }^{22}$ Department of Emergency Medicine, Medical University of Gdansk, Gdansk, Poland; ${ }^{23}$ Department of Psychiatry and Behavioral Sciences, Baylor College of Medicine, Houston, TX, USA; ${ }^{24}$ Li Chiu Kong Family Sleep Assessment Unit, Department of Psychiatry, Faculty of Medicine, The Chinese University of Hong Kong, Hong Kong SAR, People's Republic of China; ${ }^{25}$ Brain Institute, Federal University of Rio Grande do Norte, Natal, RN, Brazil; ${ }^{26}$ Physiology and Behaviour Department, Federal University of Rio Grande do Norte, Natal, RN, Brazil; ${ }^{27}$ Onofre Lopes University Hospital, Federal University of Rio Grande do Norte, Natal, RN, Brazil; ${ }^{28}$ Department of Somnology, Tokyo Medical University, Tokyo, Japan; ${ }^{29}$ Helsinki Sleep Clinic Vitalmed Research Center, Terveystalo Biobank and Research, Helsinki, Finland; ${ }^{30}$ Department of Neurosciences, Clinicum, University of Helsinki, Helsinki, Finland; ${ }^{3 /}$ Sleep Science Laboratory, Department of Neuroscience, Uppsala University, Uppsala, Sweden;

${ }^{32}$ Department of Global Public Health and Primary Care, University of Bergen, Bergen, Norway; ${ }^{33}$ Norwegian Competence Center for Sleep Disorders, Haukeland University Hospital, Bergen, Norway; ${ }^{34}$ Department of Medicine, Division of Endocrinology, Metabolism, and Molecular Medicine, Feinberg School of Medicine, Northwestern University, Chicago, IL, USA
Correspondence: Jonathan Cedernaes Email jonathan.cedernaes@medsci.uu. pandemic. Younger subjects shifted their sleep timing more during the COVID-19 pandemic. $^{1,4,7}$

Those who started to work from home or who were unemployed/laid-off during the COVID-19 pandemic also shifted their sleep timing to a later timepoint (as well as their chronotype, as measured by the midpoint of sleep on free days corrected for sleep debt on workdays - MSFsc). Overall, sleep duration seems to have increased across countries on working days, whereas its timing has been delayed during the early part of the pandemic. ${ }^{2-7}$

Even though SJL and sleep duration have improved during the early part of the COVID-19 pandemic, some studies have found evidence for reduced sleep quality. ${ }^{3,5,6,8}$ More detailed analyses of the underlying relationship remains to be carried out, especially as later bedtimes associated with the COVID-19 pandemic may also promote more negative components of mood, which typically is at its lowest point later in the night; relatedly, insomnia and depression are intimately intertwined. ${ }^{9,10}$ Some studies have reported that SJL is inversely associated with sleep quality, ${ }^{1,12}$ but the findings so far are inconsistent. ${ }^{13}$ In a larger German study, the evening-oriented chronotype (predicted by MSFsc) was negatively correlated with sleep quality and mental wellbeing. ${ }^{12}$ Other findings suggest that SJL may not be related to depressive symptoms, even in a relatively large set of healthy controls. ${ }^{14}$ However, such analyses have not been able to assess the dynamics in such associations, given that SJL and sleep problems (such as symptoms of insomnia) have not been assessed over time or in response to stressors. Furthermore, the lack of robust correlations between SJL and parameters such as wellbeing and mental health, may be due to heterogeneous samples that vary according to geographical sampling site, and that lack the ability to take agespecific characteristics into account. ${ }^{15}$ Thus, in spite of the large proportion of society that may experience significant ( $\geq 2$ hours) social jetlag on a weekly basis, ${ }^{16}$ studies are currently lacking to ascertain the possible relationship between SJL and clinically relevant sleep problems.

The COVID-19 pandemic presents a unique global, large-scale societal shift across many facets of life, for examining long-term changes in sleep habits, circadian timing of sleep, and the relation to sleep problems. In particular, studies are warranted to examine changes in symptoms of insomnia - the most frequent sleep disorder - which may have increased in prevalence during the COVID-19 pandemic. ${ }^{17}$ Herein, we sought to investigate the relationship between changes in social jetlag amid versus before the COVID-19 pandemic, and the risk of selfreported symptoms of insomnia. Our hypothesis was that a) those reporting a lower absolute level of SJL, and b) those exhibiting a notable reduction in SJL as a result of the pandemic, would have a reduced likelihood of reporting symptoms of insomnia.

\section{Materials and Methods}

The International COVID-19 Sleep Study (ICOSS) is a collaboration that assesses a variety of sleep aspects to evaluate different types of sleep-wake disorders and other symptoms affected by prolonged social confinement. The detailed protocol has previously been described. ${ }^{18}$ This research was conducted according to the Declaration of Helsinki, and all countries obtained ethical approval or exemptions 
in keeping with national research governance and regulations (see more details in Table S1). Data collection occurred between 01st May 2020 and 07th September 2020 in several places across the world (ie Austria, Canada, China (Jilin and Hong Kong, in the northeastern and southeastern regions of China, respectively), Finland, France, Germany, Italy, Japan, Norway, Poland, Sweden, United Kingdom, and United States of America) with the survey being translated according to each region's main language. For the present analysis, we assessed sleep timing reports to calculate SJL as the difference in sleep midpoint (ie, the clock time between sleep onset and waking up) on work vs free days, before and during the pandemic. Differences between SJL before and during were also computed and were used to categorize our cohort into three groups: "Unchanged" (respondents with no changes in SJL), "Increased" (respondents with positive changes in SJL), and "Reduced" (respondents with negative changes in SJL).

Data were first filtered to exclude respondents who did not answer any item of the evaluated questions (eg, sleep time and sleep-related problems), extreme sleep durations (threshold defined according to the interquartile rule with constant equal 2 - respondents that sleep less than 3 hours or more than 13 hours), and erroneous responses (eg, bedtime occurred only a few hours later than wake-up time). The final filtering resulted in a $42.56 \%$ reduction of the data set and included data from 14 regions (see Table S2).

The US cohort was the only one where participants were reimbursed to answer the questionnaire. To determine whether this introduced bias, a sensitivity analysis was performed with and without the US cohort, for the logistic regressions. Germany's cohort was excluded for the entire analysis due to a delayed data collection in comparison to other regions. The Swedish cohort was excluded from the analysis for the Basic Nordic Sleep Questionnaire analysis, due to missing data for one of the subquestions.

Before the analysis, data were weighted by sex to equalize the proportion of respondents from both sexes, for each region. Respondent's diurnal preference was also assessed via a simplified chronotype question ("Are you a morning- or evening-type person?") that classifies subjects into one of five categories: definitively morning person, more morning than evening person (moderate morning type), neither morning or evening person (intermediate type), more evening than morning person (moderate evening type), and definitively evening person. ${ }^{19}$ To complement this diurnal preference evaluation of our SJL shift groups, we also inferred chronotype by the standardized measure "midsleep on free days sleep corrected" (MSFsc). ${ }^{20}$ The MSFsc is a measure based on the sleep midpoint on free days adjusted for the sleep deficit accumulated on previous workdays. The sleep deficit is calculated as half of the difference between sleep duration on free days and the averaged sleep duration across the whole week (for more details see). ${ }^{20}$

Insomnia status was assessed by two different psychometric instruments: the Basic Nordic Sleep Questionnaire (BNSQ) and the Insomnia Severity Index (ISI). The BNSQ has been extensively validated to evaluate a variety of sleep complaints, focusing on their prevalence, incidence, and severity. ${ }^{21}$ Given that we obtained responses to BNSQ for values corresponding to both before ("pre-pandemic") and during ("pandemic") the pandemic, we could analyze how this variable changed across time ("Shift"). We focused on specific BNSQ items in order to examine, in relation to our overall hypothesis, how changes in SJL were associated with the frequency of insomnia symptoms occurring in response to the COVID-19 pandemic. Specifically, we selected three items of the BNSQ (frequency of sleep onset, sleep maintenance, and early awakening problems), to evaluate and score the respondent's symptoms of insomnia before and during the pandemic. ${ }^{22}$ The ISI instrument was additionally used to assess the severity of respondent's current sleep problems, such as the difficulty to falling and staying asleep, waking up too early in the morning, as well as the respondent's sleep dissatisfaction, difficulties with daytime functioning, noticeability of sleep problems by others, and distress caused by sleep problems during the last two weeks prior to the survey. ${ }^{23}$ All items from both the BNSQ and ISI were rated using a 5-point Likert scale (eg, for the BNSQ: $0=$ never experienced, $4=$ daily; for ISI: $0=$ no problems, $4=$ very severe problems). The total scores ranged from 0 to 12 , and from 0 to 28 , for the BNSQ and ISI instruments, respectively. Additionally, Cronbach's alpha was also calculated to measure the sub-items' internal consistency for the BNSQ (three items) and ISI (seven items) in our survey. Correlations between BNSQ and ISI scores were performed, followed by Fisher's z-transformation to look for discrepancies across regions.

We used logistic multivariate regression analysis to assess the relationship between our three SJL groups and the frequency, or severity of, symptoms of insomnia (as assessed via the BNSQ and ISI instruments, 
respectively). Responses to the BNSQ and the ISI were dichotomized as binary categories and used as the main outcome (dependent variables) to assess how the change in SJL (main predictor) in response to the COVID-19 pandemic was correlated with the risk of having symptoms of insomnia at the time of answering the questionnaire. For that, we categorized values for the BNSQ during the pandemic, as "Sporadic" (total sum scores below or equal to 6 points) or "Recurrent" (total sum scores higher than 6 points) insomnia symptoms groups. The same rationale was used for ISI scores: subjects were categorized as belonging to "Mild" (for total sum scores below or equal to 14 points) or "Moderately severe" (total sum scores higher than 14 points) groups in terms of symptoms of insomnia. The regression analyses were first run as an unadjusted model, and then in a model adjusted for sex, age at the time of the survey, SJL before the pandemic, educational level, and the extent of reported social restriction. Educational level and social restriction were rated as a 6-point Likert scale. Educational level ranged from 1 to 6 where $1=$ primary or lower secondary education, $2=$ secondary education or high school, 3 = vocational training after leaving school, $4=$ university bachelor's or equivalent level, $5=$ master's or equivalent level, and $6=$ doctoral or equivalent level. Social restriction instead ranged from 0 to 5 where $0=$ no restriction, $1=$ two weeks or less, $2=3$ to 4 weeks, $3=5$ to 6 weeks, $4=7$ to 8 weeks, and $5=$ more than 8 weeks. We additionally controlled our analysis for daylength, country-specific COVID-19 severity and the reported level of psychological distress. Daylength was calculated considering the median date of the survey collection period in each region. The country-specific COVID-19 severity was evaluated as the cumulative sum of cases (at the moment of the survey) per one hundred thousand inhabitants for each region. We decided to use this variable since the temporal dynamics in the number of cases and deaths among regions can negatively impact mood, and, consequently, sleep quality of respondents. ${ }^{24}$ Psychological distress was measured through the Patient Health Questionnaire for Depression and Anxiety (PHQ-4), a brief screening scale which merges two subscales for evaluation of depression (GAD-2) and anxiety (PHQ-2). The total sum of those subscales range from 0 to 12 and has been found to be significantly associated with respondent's perception of distress. ${ }^{25,26}$ Regression results are presented as odds ratio (OR) and respective
95\% confidence interval (CI) (ie, OR [lower and upper limit]).

Non-parametric tests like Wilcoxon signed-rank test, Mann-Whitney's $U$-test, and the Kruskal-Wallis $H$-test were used to account for the assumption of non-normally distributed data. Significance was set at $\mathrm{P}<0.05$ and reported as two-sided P-values unless specified otherwise. Pearson's chi-squared test and adjusted residuals were computed to evaluate if sex or social restriction proportion differences did not arise by chance. A locally weighted regression (ie, non-parametric, locally estimated scatterplot smoothing - LOESS - regression) was also implemented to understand and reveal trends between SJL parameters before and during the pandemic. Neighborhood size was controlled and adjusted to $95 \%$ of the entire data for the locally weighted regression. All statistical analyses, variable computation, and graphs were done using the $\mathrm{R}$ software ( $\mathrm{v}$ 4.0.3), with the packages survey $^{27}$ (v 4.0), DescTools (v 0.99.42) and the ggplot $2^{28}$ (v 3.3.3). Group comparison results are presented as median and interquartile range (ie, median [1st, 3rd interquartile]), or as mean and standard deviation as detailed for each section.

\section{Results}

\section{Differences in SJL During vs Before the COVID-19 Pandemic}

Based on their sleep midpoint on weekends vs free days, respondents indicated that they overall had significantly reduced SJL during compared with before the COVID-19 pandemic (SJL before: 00:59 \pm 01:10; during: 00:41 \pm 01:07 hours, Figure 1A). Using local polynomial regression, we observed that, in general, respondents who reported pre-pandemic SJL values that exceeded $19 \mathrm{~min}$, were those who exhibited a decrease in SJL during the pandemic (Figure 1C). Of note, whereas the majority of respondents $(46 \%)$ thus reported a reduction in $\mathrm{SJL}$ $(-00: 58 \pm 00: 56$ hours) in response to the COVID-19 pandemic, nearly a third (34\%) reported no change in SJL, and 20\% reported increased SJL (00:44 \pm 01:01 hours) (Figure 1B).

\section{SJL Shift Groups and Their Particular Characteristics}

As summarized in Table 1, compared with the unchanged SJL group, subjects who reported either an increase or decrease in their SJL in response to the 

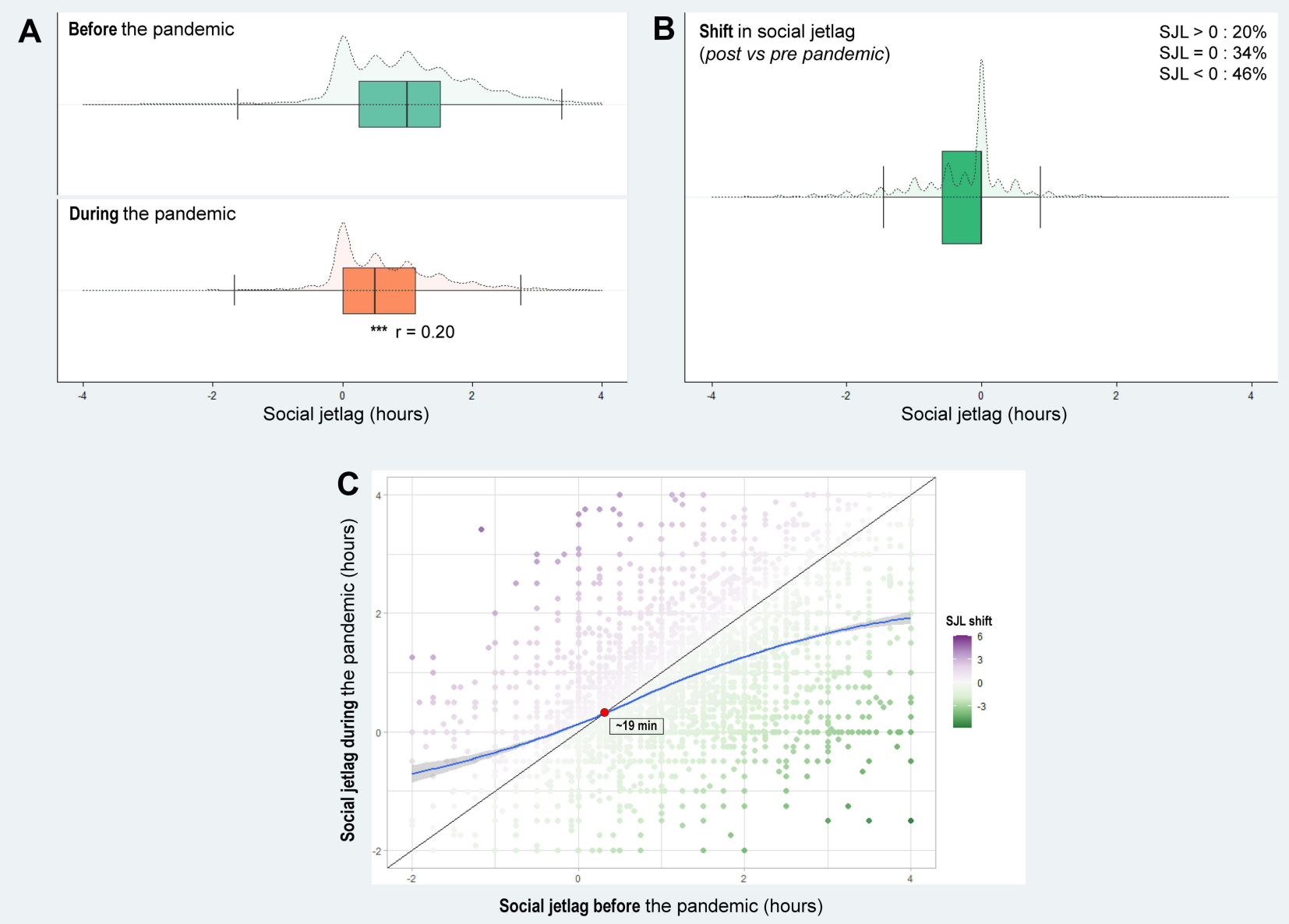

Figure I Difference in social jetlag before and during the COVID-19 pandemic in the studied 14 regions.

Notes: (A) Social jetlag before and during the COVID-19 pandemic; (B) Social jetlag change. Boxplots (solid lines) illustrate the median and interquartiles; whereas violin plots (dashed lines) illustrate the data distribution; (C) Scatterplot of social jetlag before ( $x$-axis) versus during ( $y$-axis) the pandemic. The change in social jetlag is represented by the plot colors purple, green and white for positive, negative and no change, respectively; LOESS smoothed line in blue; No change in SJL also represented by the diagonal black line; the orange circle indicates the intercept between LOESS regression and diagonal line. $* * * P<0.00$ I, Wilcoxon signed-rank test; $r$ : effect size.

pandemic, exhibited significantly greater SJL before the pandemic (indicating reduced regularity in sleep timing). They were also more often women, younger, more often reported having a university education, had experienced more weeks in confinement, and were geographically located in regions with longer photophase (ie, period of light during a day-night cycle) at the time of the survey, compared with subjects from the unchanged SJL group. Those who reported later MSFsc values either before or during the pandemic - or lived in regions where the COVID-19 pandemic was more severe, as well as those who reported more psychological distress, were significantly more prevalent in the increased and reduced SJL groups, compared with the unchanged SJL group (Table 1). The later MSFsc values in the two shifted SJL groups, was also reflected by descriptively more subjects with evening chronotypes (ie, moderate and definitively evening types) in those two groups, compared with the unchanged SJL group.

Differences in sleep timing were also observed between the three SJL groups across the pandemic. Before the pandemic, respondents from the increased and reduced SJL groups reported significantly later waking times and longer sleep durations than the unchanged group during work and free days. In this same prepandemic period, respondents from the reduced SJL group went to bed and woke up significantly later than both unchanged and increased SJL groups during free days.

In response to the pandemic, individuals in the increased and reduced SJL groups still reported significantly later waking times and longer sleep durations when 
Table I Main Characteristics of Respondents Within Different Social Jetlag Categories

\begin{tabular}{|c|c|c|c|c|c|c|}
\hline & \multicolumn{6}{|c|}{ Social Jetlag Shift Categories } \\
\hline & \multicolumn{2}{|c|}{ Unchanged } & \multicolumn{2}{|c|}{ Increased } & \multicolumn{2}{|c|}{ Reduced } \\
\hline Number of Respondents & \multicolumn{2}{|c|}{6555} & \multicolumn{2}{|c|}{3804} & \multicolumn{2}{|c|}{8811} \\
\hline General characteristics & & $95 \% \mathrm{Cl}$ & & $95 \% \mathrm{Cl}$ & & $95 \% \mathrm{Cl}$ \\
\hline$\%$ Men & $56.3^{\mathrm{a}}$ & - & $48.0^{\mathrm{a}}$ & - & $46.2^{\mathrm{a}}$ & - \\
\hline \% Women & $43.7^{\mathrm{a}}$ & - & $52.0^{\mathrm{a}}$ & - & $53.8^{\mathrm{a}}$ & - \\
\hline \% Definitively Morning-types & 19.7 & - & I7.I & - & 13.3 & - \\
\hline \% Moderate Morning-types & 22.3 & - & 20.8 & - & 22.6 & - \\
\hline$\%$ Intermediate-types & 27.9 & - & 23.3 & - & 21.3 & - \\
\hline \% Moderate Evening-types & 20.5 & - & 26.3 & - & 27.6 & - \\
\hline \% Definitively Evening-types & 9.5 & - & 12.5 & - & 15.2 & - \\
\hline$\%$ Subjects reporting social restriction & $36.1^{\mathrm{a}}$ & - & $51.3^{\mathrm{a}}$ & - & $57.3^{\mathrm{a}}$ & - \\
\hline Social restriction level & 0 & $(0,2)$ & $\mathrm{I}^{\mathrm{b}}$ & $(0,4)$ & $I^{b, c}$ & $(0,5)$ \\
\hline Age at the time of the survey (years) & 42 & $(30,56)$ & $36^{\mathrm{b}}$ & $(26,49)$ & $35^{\mathrm{b}, \mathrm{c}}$ & $(25,47)$ \\
\hline Educational level at the time of the survey ${ }^{\dagger}$ & 4 & $(3,5)$ & $4^{\mathrm{b}}$ & $(3,5)$ & $4^{\mathrm{b}}$ & $(3,5)$ \\
\hline Daylength (hours) at the time of the survey & 15.2 & $(14.6,16.1)$ & $15.3^{\mathrm{b}}$ & $(14.6,16.1)$ & $15.3^{b, c}$ & $(14.6,16.2)$ \\
\hline Country-specific COVID-19 severity & 117 & $(14,239)$ & $228^{\mathrm{b}}$ & $(14,425)$ & $228^{\mathrm{b}, \mathrm{c}}$ & $(117,425)$ \\
\hline Symptoms of Insomnia & & $95 \% \mathrm{Cl}$ & & $95 \% \mathrm{Cl}$ & & $95 \% \mathrm{Cl}$ \\
\hline BNSQ before the pandemic & 2 & $(0,4)$ & $3^{\mathrm{b}}$ & $(I, 5)$ & $3^{\mathrm{b}}$ & $(I, 5)$ \\
\hline BNSQ during the pandemic & 2 & $(0,5)$ & $4^{\mathrm{b}}$ & $(I, 7)$ & $4^{\mathrm{b}}$ & $(I, 7)$ \\
\hline BNSQ change (during - before) & 0 & $(0,0)$ & $0^{\mathrm{b}}$ & $(0,2)$ & $0^{b, c}$ & $(0,3)$ \\
\hline ISI during the pandemic & 5 & $(2,10)$ & $8^{\mathrm{b}}$ & $(4,13)$ & $8^{\mathrm{b}}$ & $(4,13)$ \\
\hline Psychological distress & & $95 \% \mathrm{Cl}$ & & $95 \% \mathrm{Cl}$ & & $95 \% \mathrm{Cl}$ \\
\hline PHQ-2 (depression component) score & 0 & $(0,2)$ & $2^{b}$ & $(0,2)$ & $2^{b, c}$ & $(0,3)$ \\
\hline GAD-2 (anxiety component) score & I & $(0,2)$ & $2^{\mathrm{b}}$ & $(0,3)$ & $2^{b, c}$ & $(0,3)$ \\
\hline PHQ-4 total score & 1 & $(0,4)$ & $3^{\mathrm{b}}$ & $(1,5)$ & $3^{\mathrm{b}, \mathrm{c}}$ & $(1,6)$ \\
\hline Social jetlag (hh:mm) & & SD & & SD & & SD \\
\hline Before the pandemic & $00: 38$ & $(00: 58)$ & $00: 35$ & $(01: 21)$ & $01: 25^{\mathrm{b}, \mathrm{c}}$ & $(01: 05)$ \\
\hline During the pandemic & $00: 38$ & $(00: 58)$ & $01: 19^{b}$ & $(01: 20)$ & $00: 27^{\mathrm{b}, \mathrm{c}}$ & $(00: 59)$ \\
\hline Change (during - before) & 00:00 & $(00: 00)$ & $00: 44^{\mathrm{b}}$ & $(01: 01)$ & $-00: 58^{b, c}$ & $(00: 56)$ \\
\hline MSFsc before the pandemic & 03:39 & $(01: 35)$ & $03: 49^{b}$ & $(01: 35)$ & $04: 23^{\mathrm{b}, \mathrm{c}}$ & $(01: 29)$ \\
\hline MSFsc during the pandemic & 03:45 & $(01: 47)$ & $04: 26^{\mathrm{b}}$ & $(01: 57)$ & $04: 27^{\mathrm{b}}$ & $(01: 56)$ \\
\hline Sleep timing (hh:mm) & & SD & & SD & & SD \\
\hline Bedtime on workdays (before the pandemic) & $23: 21$ & $(01: 32)$ & $23: 29$ & $(01: 37)$ & $23: 15^{\mathrm{b}, \mathrm{c}}$ & $(01: 22)$ \\
\hline Waking time on workdays (before the pandemic) & $06: 44$ & $(01: 30)$ & $07: 04^{b}$ & $(01: 38)$ & $06: 47^{b, c}$ & $(01: 19)$ \\
\hline Sleep duration on workdays (before the pandemic) & 07:23 & $(01: 17)$ & $07: 35^{\mathrm{b}}$ & $(01: 14)$ & $07: 32^{\mathrm{b}}$ & $(01: 16)$ \\
\hline Bedtime on free days (before the pandemic) & $23: 40$ & $(01: 34)$ & $23: 37$ & $(01: 45)$ & $00: 06^{\mathrm{b}, \mathrm{c}}$ & $(01: 33)$ \\
\hline Waking time on free days (before the pandemic) & 07:39 & $(01: 47)$ & $08: 06^{b}$ & $(01: 46)$ & $08: 45^{b, c}$ & $(0 I: 42)$ \\
\hline Sleep duration on free days (before the pandemic) & 07:58 & $(01: 26)$ & $08: 28^{b}$ & $(01: 24)$ & $08: 38^{\mathrm{b}, \mathrm{c}}$ & $(01: 24)$ \\
\hline Bedtime on workdays (during the pandemic) & $23: 25$ & $(01: 37)$ & $23: 20^{b}$ & $(01: 46)$ & $00: 03^{b, c}$ & $(01: 54)$ \\
\hline Waking time on workdays (during the pandemic) & 06:48 & $(01: 39)$ & $06: 56^{\mathrm{b}}$ & $(0 I: 4 I)$ & $07: 56^{\mathrm{b}, \mathrm{c}}$ & $(02: 05)$ \\
\hline Sleep duration on workdays (during the pandemic) & 07:22 & $(01: 25)$ & $07: 35^{\mathrm{b}}$ & $(01: 28)$ & $07: 50^{b, c}$ & $(01: 33)$ \\
\hline Bedtime on free days (during the pandemic) & $23: 46$ & $(01: 39)$ & $00: 12^{b}$ & $(01: 56)$ & $00: 16^{\mathrm{b}}$ & $(01: 59)$ \\
\hline Waking time on free days (during the pandemic) & 07:42 & $(01: 55)$ & $08: 39^{b}$ & $(02: 07)$ & $08: 38^{b}$ & $(02: 08)$ \\
\hline Sleep duration on free days (during the pandemic) & 07:55 & $(0 \mathrm{I}: 3 \mathrm{I})$ & $08: 25^{\mathrm{b}}$ & $(01: 34)$ & $08: 20^{\mathrm{b}}$ & $(01: 37)$ \\
\hline
\end{tabular}

Notes: General characteristics and symptoms of insomnia are presented as percentage or median and interquartile range. Social jetlag and sleep timing are presented as mean and standard deviation. Data were weighted by sex to equalize the proportion of respondents from both sexes, for each region. ${ }^{\dagger} \mathrm{I}=$ primary or lower secondary education, 2 = secondary education or high school, 3 = Vocational training after leaving school, $4=$ university bachelor's or equivalent level, $5=$ master's or equivalent level, and $6=$ doctoral or equivalent level. ${ }^{a} P<0.00 I$, for Pearson's chi-squared test, followed by adjusted residual analysis, significant differences across the three groups. ${ }^{b} P<0.00 \mathrm{I}$, for Mann-Whitney's U-test comparing Increased or Reduced vs Unchanged group. ' $P<0.00$ I, for Mann-Whitney's U-test comparing Increased vs Reduced.

Abbreviations: BNSQ, Basic Nordic Sleep Questionnaire; GAD-2, Generalized Anxiety Disorder 2-item; ISI, Insomnia Severity Index; MSFsc, midsleep on free days sleep corrected; PHQ-2, Patient Health Questionnaire-2; PHQ-4, Patient Health Questionnaire-4; SD, standard deviation; 95\% Cl, 95\% confidence interval. 
compared with the unchanged group, during both workdays and free days. Notably, during workdays, respondents from the reduced SJL group delayed their bedtime significantly more, whereas the increased SJL group advanced their sleep timing, compared with the unchanged group (Table 1). Noteworthy, both the increased and reduced SJL groups exhibited later MSFsc, before as well as during the pandemic, compared with the unchanged group.

Both the BSNQ and ISI instruments revealed high internal consistency (Cronbach's alpha for BNSQ and ISI equal to 0.86 and 0.90 , respectively). The responses to these instruments were furthermore highly correlated across all countries, with similar correlations across the different countries (and translations), as determined by Fisher Z-scores (Table S3). When exploring the differences in the frequency of insomnia symptoms between the different SJL groups, we observed that subjects who had either reduced or increased their SJL, reported higher scores for the BNSQ (indicating more frequent episodes of insomnia) compared with those who showed no changes as a result of the pandemic (Table 2 and Figure 2A and B). Regarding the severity of the symptoms of insomnia (ie, the ISI scores), respondents with increased or reduced SJL reported more severe symptoms of insomnia compared with the unchanged group (Table 2 and Figure 2C). These associations (both for the BNSQ and ISI) were somewhat attenuated in our adjusted model (adjusted for sex and age at the time of the survey, SJL before the pandemic, educational level, social restriction, country-specific Covid severity and psychological distress), but still remained significant. Furthermore, our adjusted models improved our goodness-of-fit (as reflected by higher Pseudo- $\mathrm{R}^{2}$ values, BNSQ: unadjusted $=0.015$, adjusted $=0.233$; ISI: unadjusted $=0.025$, adjusted $=0.277$; Table 2). A full description of the unadjusted odds ratios for all covariates is listed in Table S4.

A subsequent sensitivity analysis did not show significant differences in either logistic regression odds ratios or fitness - for the association between SJL group and symptoms of insomnia - after removal of the US cohort (Table S5), or after processing data without weighting for respondents' sex (Table S6).

\section{Discussion}

As a result of the COVID-19 pandemic, respondents in our multinational survey reduced the differences in sleep timing between workdays and free days - ie, their social jetlag (SJL) - by almost 18 minutes. However, subjects with either increased or reduced SJL in response to the pandemic had between $23 \%$ and $54 \%$ higher odds ratio of experiencing more frequent or severe symptoms of insomnia, compared with those who did not report any change in their SJL.

Despite not having directly evaluated either severity or frequency of symptoms of insomnia, other studies have associated the pandemic-associated reduction in SJL with worse sleep quality. ${ }^{3,5,6,8}$ In a survey of subjects from Austria, Germany and Switzerland, Blume et $\mathrm{al}^{5}$ observed reduced SJL as well as a slight reduction in their self-reported sleep quality, increased subjective burden and lower mental

Table 2 Logistic Regression of the Association of Insomnia Severity and the Presence of a Reported Shift in the Amount of Social Jetlag from Before to During the Early Part of the COVID-19 Pandemic in 2020

\begin{tabular}{|c|c|c|c|c|c|c|}
\hline & \multirow{2}{*}{$\begin{array}{c}\text { Unadjusted } \\
\text { OR }\end{array}$} & \multicolumn{2}{|c|}{$95 \% \mathrm{Cl}$} & \multirow[t]{2}{*}{ Adjusted OR } & \multicolumn{2}{|c|}{$95 \% \mathrm{Cl}$} \\
\hline & & Lower & Upper & & Lower & Upper \\
\hline \multicolumn{7}{|c|}{ Recurrent insomnia (defined as a BNSQ score $>6$ ) } \\
\hline Unchanged SJL & 1.00 & 1.00 & 1.00 & 1.00 & 1.00 & 1.00 \\
\hline Increased SJL & 1.59 & 1.39 & 1.83 & 1.23 & 1.05 & 1.44 \\
\hline Reduced SJL & 1.70 & 1.52 & 1.90 & 1.27 & 1.11 & 1.46 \\
\hline \multicolumn{7}{|c|}{ Moderately severe insomnia (defined as an ISI score $>\mid 4$ ) } \\
\hline Unchanged SJL & 1.00 & 1.00 & 1.00 & 1.00 & 1.00 & 1.00 \\
\hline Increased SJL & 1.98 & 1.70 & 2.31 & 1.54 & 1.28 & 1.86 \\
\hline Reduced SJL & 2.16 & 1.90 & 2.46 & 1.54 & 1.31 & 1.81 \\
\hline
\end{tabular}

Notes: Adjusted odds ratios (ORs) were controlled for respondents' sex and age at the time of the survey, SJL before the pandemic, educational level, social restriction, country-specific covid severity and psychological distress. We additionally controlled for the length of the day when the respondent filled out the survey. Unchanged SJL group was settled as the reference. Pseudo- $\mathrm{R}^{2}$ (Nagelkerke method) for non-adjusted and adjusted models: BNSQ: 0.015 and 0.234 ; ISI: 0.025 and 0.277 , respectively. Abbreviations: BNSQ, Basic Nordic Sleep Questionnaire; ISI, Insomnia Severity Index; SJL, social jetlag; OR, odds ratio; $95 \%$ Cl, $95 \%$ confidence interval. 

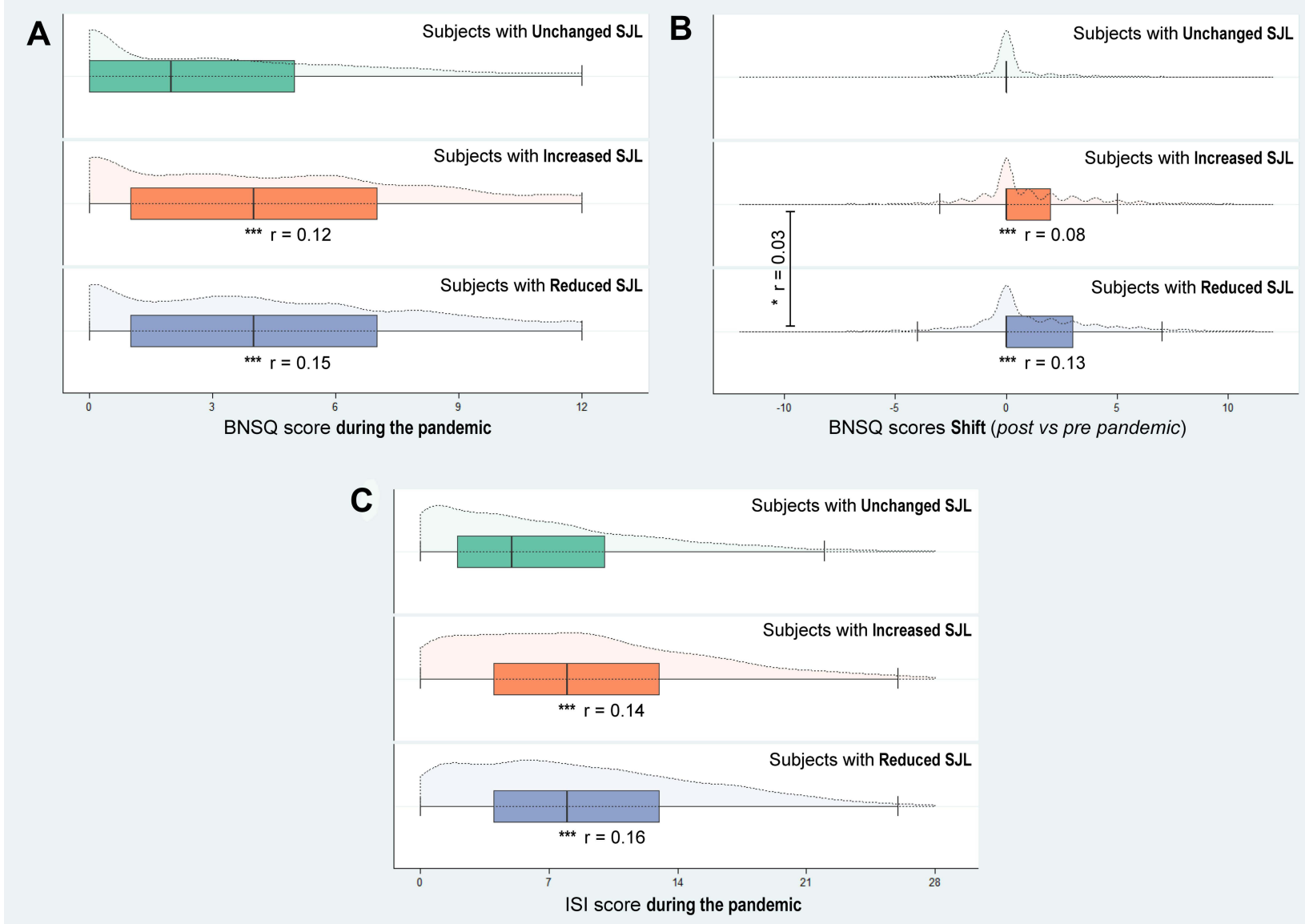

Figure 2 Insomnia status of the respondents from the different social jetlag groups (ie, Unchanged, Increased and Reduced) in response to the COVID-I9 pandemic. Notes: (A) BNSQ scores during the pandemic; (B) Change in BNSQ scores; (C) ISI scores during the pandemic. Boxplots (solid lines) illustrate the median and interquartiles, whereas the violin plots (dashed lines) illustrate the data distribution. $* P<0.05$, $* * * P<0.00$ I, Mann-Whitney's $U$-test. $r$ : effect size.

and physical wellbeing, during the pandemic. Based on our analysis, we advocate for a more nuanced analysis of how the pandemic may have affected sleep in humans.

A critical aspect for considering factors that contribute to the risk and long-term changes in the prevalence of symptoms of insomnia, is not only the amount of SJL, but also how sleep/wake rhythms are temporally aligned to the external light-dark cycle. Typically, positive components of mood peak in the morning whereas the negative components peak at circadian night. ${ }^{9,10}$ Herein we observed that participants also shifted their bedtime to later hours - an effect mainly observed during workdays, as previously reported, to be more in line with the weekend timing. Thus, even though a reduction in SJL may, in general, be considered a positive feature, a shift to later bedtimes may possibly have resulted in an increase in negative components of mood, and thereby explain the observed increase in both the frequency and severity of insomnia even in the group that exhibited a reduced SJL in response to the pandemic. In essence, participants who were able to reduce their SJL may have done so at the expense of later bed- and wakeup times.

Previous studies have noted that later chronotype is associated with more pronounced symptoms of insomnia. ${ }^{29,30}$ This may be due to altered brain metabolism in the prefrontal cortex and striatum. ${ }^{31}$ An overall shift during the pandemic toward an evening preference represented by a delayed sleep schedule - may represent an exogenous mechanism by which worsened mood and sleep arise by increasing the desynchrony between a more delayed sleep schedule and the external light-dark cycle, which is still dictated by solar time.

Maintaining a regular sleep schedule with regular bed and wake times is important for achieving adequate sleep. 
Importantly, greater variability in bed and wake time has been observed in people with insomnia. ${ }^{32-34}$ This may explain why subjects who reported increased SJL in response to the pandemic, may have exhibited higher odds of experiencing more frequent and severe episodes of insomnia, compared with the group that did not exhibit a change in SJL. In this context, it is important to highlight that many in both the increased and reduced SJL groups already presented recurrent symptoms of insomnia before the pandemic, and that these symptoms worsened during the pandemic. Morin et $\mathrm{al}^{35}$ reported from a large cohort, evaluated during five consecutive years, that subjects with higher baseline insomnia had a greater risk of persistent sleep disturbances and persisting insomnia. It would therefore be of clinical relevance to be able to follow-up how persistent or plastic the increase in sleep issues in certain groups are in response to the pandemic, and whether a shift to earlier bedtimes may alleviate some of the observations made herein in those with altered SJL.

Our findings also shows that those who reported changes in SJL in response to the pandemic, also had experienced more and longer restrictions related to the COVID-19 pandemic. On the positive side, this may have allowed for greater flexibility in their daily social schedules (eg, by being able to work from home, having reduced working hours). On the negative side, such societal restrictions may add stress that could at least partially account for the increased symptoms of insomnia found herein, in those who were able to shift their sleep/wake habits to be less different in timing between free and working days. Thus, such stress may possibly partially negate the possible beneficial impact of reduced sleep timing variability.

Moreover, recent studies have found that the COVID19 pandemic may have increased the incidence of impaired mood and sleep. ${ }^{17,36,37}$ In two Italian cohort studies, ${ }^{6,8}$ decreased/poor sleep quality has been observed to be associated with a higher prevalence of symptoms of depression, anxiety, and stress. In this sense, despite canonically considering a reduction in $\mathrm{SJL}$ as a marker of improved sleep and well-being, ${ }^{12}$ the psychological burden associated with the COVID-19 pandemic, could in sum explain why even reduced levels of SJL - at least as herein early in the pandemic - were associated with worse symptoms of insomnia and sleep quality. Our data corroborate these previous findings, since we find that those with changes in the extent of social jetlag reported higher levels of psychological distress. Another factor that could contribute to this type of sleep irregularity and impaired sleep quality is an increase in the use of digital media, a parameter which we did not assess. Others have however noted that during the pandemic, use of digital media near bedtime has increased across all age groups. ${ }^{1,6}$ Furthermore, it is known that light at night, especially blue light that often dominates the light spectrum emitted by electronic devices, may contribute to disrupted evening melatonin secretion, which may promote delayed sleep onset and impaired objective sleep parameters. ${ }^{38,39}$

An overall decrease in SJL as a result of the COVID19 pandemic, has been observed in prior studies. ${ }^{2-7,40}$ In an Argentinian cohort, Leone et $\mathrm{al}^{4}$ reported that subjects slept longer and later during lockdown (on weekdays), exhibiting lower levels of SJL (decreased by 54 minutes). In another international survey across 40 countries, Korman et $\mathrm{al}^{2}$ reported an overall reduction in SJL of 30 minutes. In the US, Wright et $\mathrm{al}^{7}$ reported a SJL reduction of 18 minutes in a young cohort of university students. Another recent study from the US, authored by Rezaei and Grandner, ${ }^{40}$ highlighted an overall increase in sleep duration and delayed sleep phase (mainly during workdays), which contributed to a reduction in SJL across all age groups and both sexes. Our present study results build upon these prior findings, but also add a clinically relevant sleep aspect that may be worth considering when evaluating changes in SJL.

\section{Strengths and Limitations}

Several strengths apply to our study. Our findings are robust to adjustment for important confounders, such as daylight length, which affects sleep timing, ${ }^{41}$ and that we report data from countries spanning several continents. Another strength is that our findings were highly consistent across two widely used insomnia scales, regarding the association of changes in SJL and symptoms of insomnia.

Despite these strengths, several limitations apply. We did not have access to data reported over several days, and our one-timepoint data are as such more prone to recall bias. Moreover, varying response rate between survey sites, after data filtering, may have impacted the extent of which our results are generalizable across the studied regions. We also lack more accurate methods to ascertain sleep midpoint, such as sleep determined by actigraphy or polysomnography. Such data could also have helped to inform us about the timing of physical activity and environmental light exposure, which may have modified our results, as reduced physical activity 
may also have contributed to impaired mood during the COVID-19 pandemic. $^{37}$ Sleep timing can indeed be greatly influenced by 24-h light exposure at the individual level, a parameter that is hard to assess from questionnaire data. While we tried to adjust for daylength around the time of the survey, we cannot rule out that some of our results - like all results based on recall spanning recent months - could be prone to bias due to seasonal changes. This may be of greater relevance in more northerly or southerly regions where temperature, humidity and daylength vary considerably across the seasons.

\section{Conclusion}

Our findings reveal how changes in social jetlag may be linked to the risk of reporting symptoms of insomnia. In particular, we highlight how the consistency of sleep, as reflected by changes in the degree of social jetlag, may be important to consider when determining how social jetlag compared with the actual timing of sleep - are associated with the risk of experiencing novel-onset or persistence of symptoms of insomnia, the worldwide most prevalent sleep disorder.

\section{Acknowledgments}

We like to thank Dr Fang Han from Peking University People's Hospital for the survey data collection in the Jilin region. We would like to thank all the anonymous participants for responding to our questions. The study was supported by the Swedish Society for Medical Research (SSMF), the Swedish Research Council (VR; 201901051), and the following foundations: Diabetesfonden (2019-489), Diabetes Wellness Sverige (25-2231), Selander, Göran Gustafsson, Magnus Bergvalls (201802975), NovoNordisk (NNF19OC0056694), the Swedish Brain (FO2019-0086; FO2019-0274), and The Swedish Cancer foundation. None of the study sponsors/funders were involved in the design of the study; the collection, analysis, and interpretation of data; writing the report; and they did not impose any restrictions regarding the publication of the report.

\section{Disclosure}

Professor Colin A Espie report grants from National Institutes of Health Research, personal fees from Big Health, outside the submitted work; Dr Giuseppe Plazzi report personal fees from Jazz Pharmaceuticals, personal fees from Takeda, personal fees from Bioprojet, personal fees from Idorsia, during the conduct of the study; personal fees from Jazz Pharmaceuticals, personal fees from Takeda, personal fees from Bioprojet, personal fees from Idorsia, outside the submitted work; Dr Kentaro Matsui report personal fees from Eisai, personal fees from Meiji Seika Pharma, personal fees from MSD, personal fees from Otsuka Pharmaceutical, personal fees from Takeda Pharmaceutical, personal fees from Yoshitomi Pharmaceutical, outside the submitted work; Dr Yuichi Inoue report personal fees from Eisai Co., Ltd, personal fees from Otsuka Pharmaceutical Co., Ltd, personal fees from Takeda Pharma ceutical Co., Ltd, personal fees from Astellas Pharma Inc, personal fees from MSD K.K., grants from Idorsia Pharmaceuticals Japan., Ltd, grants from Philips Japan Co., Ltd, grants from KOIKE Medical Co., Ltd, outside the submitted work; Dr Christian Benedict report grants from Repha $\mathrm{GmBH}$, outside the submitted work. The authors report no other conflicts of interest in this work.

\section{References}

1. Sinha M, Pande B, Sinha R. Impact of COVID-19 lockdown on sleep-wake schedule and associated lifestyle related behavior: a national survey. J Public Health Res. 2020;9(3):239-245. doi:10.4081/jphr.2020.1826

2. Korman M, Tkachev V, Reis C, et al. COVID-19-mandated social restrictions unveil the impact of social time pressure on sleep and body clock. Sci Rep. 2020;10(1):22225. doi:10.1038/s41598-020-79299-7

3. Raman S, Coogan AN. Effects of societal-level COVID-19 mitigation measures on the timing and quality of sleep in Ireland. Sleep Med. 2021;(January). doi:10.1016/j.sleep.2021.02.024

4. Leone MJ, Sigman M, Golombek DA. Effects of lockdown on human sleep and chronotype during the COVID-19 pandemic. Curr Biol. 2020;30(16):R930-R931. doi:10.1016/j.cub.2020.07.015

5. Blume C, Schmidt MH, Cajochen C. Effects of the COVID-19 lockdown on human sleep and rest-activity rhythms. Curr Biol. 2020;30 (14):R795-R797. doi:10.1016/j.cub.2020.06.021

6. Cellini N, Canale N, Mioni G, Costa S. Changes in sleep pattern, sense of time and digital media use during COVID-19 lockdown in Italy. J Sleep Res. 2020;29(4):1-5. doi:10.1111/jsr.13074

7. Wright KP, Linton SK, Withrow D, et al. Sleep in university students prior to and during COVID-19 stay-at-home orders. Curr Biol. 2020;30(14):R797-R798. doi:10.1016/j.cub.2020.06.022

8. Franceschini C, Musetti A, Zenesini C, et al. Poor sleep quality and its consequences on mental health during the COVID-19 lockdown in Italy. Front Psychol. 2020;11(November):1-15. doi:10.3389/ fpsyg.2020.574475

9. Golder SA, Macy MW. Diurnal and seasonal mood vary with work, sleep, and daylength across diverse cultures. Science (80-). 2011;333 (6051):1878-1881. doi:10.1126/science.1202775

10. Emens JS, Berman AM, Thosar SS, et al. Circadian rhythm in negative affect: implications for mood disorders. Psychiatry Res. 2020;293(July):113337. doi:10.1016/j.psychres.2020.113337

11. Südy ÁR, Ella K, Bódizs R, Káldi K. Association of social jetlag with sleep quality and autonomic cardiac control during sleep in young healthy men. Front Neurosci. 2019;13(September):1-10. doi:10.3389/fnins.2019.00950

12. Wittmann M, Dinich J, Merrow M, Roenneberg T. Social jetlag: misalignment of biological and social time. Chronobiol Int. 2006;23 (1-2):497-509. doi:10.1080/07420520500545979 
13. Rutters F, Lemmens SG, Adam TC, et al. Is social jetlag associated with an adverse endocrine, behavioral, and cardiovascular risk profile? J Biol Rhythms. 2014;29(5):377-383. doi:10.1177/ 0748730414550199

14. Knapen SE, Riemersma-van der Lek RF, Antypa N, Meesters Y, Penninx BWJH, Schoevers RA. Social jetlag and depression status: results obtained from the Netherlands Study of Depression and Anxiety. Chronobiol Int. 2018;35(1):1-7. doi:10.1080/ 07420528.2017 .1374966

15. Henderson SEM, Brady EM, Robertson N. Associations between social jetlag and mental health in young people: a systematic review. Chronobiol Int. 2019;36(10):1316-1333. doi:10.1080/ 07420528.2019 .1636813

16. Roenneberg T, Allebrandt KV, Merrow M, Vetter C. Social jetlag and obesity. Curr Biol. 2012;22(10):939-943. doi:10.1016/j. cub.2012.03.038

17. Pappa S, Ntella V, Giannakas T, Giannakoulis VG, Papoutsi E, Katsaounou P. Prevalence of depression, anxiety, and insomnia among healthcare workers during the COVID-19 pandemic: a systematic review and meta-analysis. Brain Behav Immun. 2020;88(January):901-907. doi:10.1016/j.bbi.2020.05.026

18. Partinen M, Bjorvatn B, Holzinger B, et al. Sleep and circadian problems during the coronavirus disease 2019 (COVID-19) pandemic: the International COVID-19 Sleep Study (ICOSS). J Sleep Res. 2021;30(1):2-9. doi:10.1111/jsr.13206

19. Knutson KL, von Schantz M. Associations between chronotype, morbidity and mortality in the UK Biobank cohort. Chronobiol Int. 2018;35(8):1-9. doi:10.1080/07420528.2018.1454458

20. Roenneberg P, Zerbini W. Chronotype and social jetlag: a (self-) critical review. Biology (Basel). 2019;8(3):54. doi:10.3390/ biology 8030054

21. Partinen M, Gislason T. Basic Nordic Sleep Questionnaire (BNSQ): a quantitated measure of subjective sleep complaints. J Sleep Res. 1995;4:150-155. doi:10.1111/j.1365-2869.1995.tb00205.x

22. Lavonius M, Railo H, Karlsson L, et al. Maternal sleep quality during pregnancy is associated with neonatal auditory ERPs. Sci Rep. 2020;10(1):7228. doi:10.1038/s41598-020-64160-8

23. Morin CM, Belleville G, Bélanger L, Ivers H. The insomnia severity index: psychometric indicators to detect insomnia cases and evaluate treatment response. Sleep. 2011;34(5):601-608. doi:10.1093/sleep/ 34.5.601

24. Simor P, Polner B, Báthori N, et al. Home confinement during the COVID-19: day-to-day associations of sleep quality with rumination, psychotic-like experiences, and somatic symptoms. Sleep. 2021;44 (301):1-27. doi:10.1093/sleep/zsab029

25. Mills SD, Fox RS, Pan TM, Malcarne VL, Roesch SC, Sadler GR. Psychometric evaluation of the patient health questionnaire-4 in Hispanic Americans. Hisp J Behav Sci. 2015;37(4):560-571. doi:10.1177/0739986315608126

26. Kroenke K, Spitzer RL, Williams JBW, Löwe B. An ultra-brief screening scale for anxiety and depression: the PHQ-4. Psychosomatics. 2009;50(6):613-621. doi:10.1016/S0033-3182(09) 70864-3
27. Lumley T. Analysis of complex survey samples. J Stat Softw. 2004;9 (8):1-9. doi:10.18637/jss.v009.i08

28. Wickham H. Ggplot2. New York: Springer; 2009. doi:10.1007/9780-387-98141-3

29. Alvaro PK, Roberts RM, Harris JK. The independent relationships between insomnia, depression, subtypes of anxiety, and chronotype during adolescence. Sleep Med. 2014;15(8):934-941. doi:10.1016/j. sleep.2014.03.019

30. Li SX, Chan NY, Man Yu MW, et al. Eveningness chronotype, insomnia symptoms, and emotional and behavioural problems in adolescents. Sleep Med. 2018;47:93-99. doi:10.1016/j. sleep.2018.03.025

31. Hasler BP, Germain A, Nofzinger EA, et al. Chronotype and diurnal patterns of positive affect and affective neural circuitry in primary insomnia. J Sleep Res. 2012;21(5):515-526. doi:10.1111/j.13652869.2012.01002.x

32. Stepanski EJ, Wyatt JK. Use of sleep hygiene in the treatment of insomnia. Sleep Med Rev. 2003;7(3):215-225. doi:10.1053/ smrv.2001.0246

33. Irish LA, Kline CE, Gunn HE, Buysse DJ, Hall MH. The role of sleep hygiene in promoting public health: a review of empirical evidence. Sleep Med Rev. 2015;22(701):23-36. doi:10.1016/j. smrv.2014.10.001

34. Buysse DJ, Cheng Y, Germain A, et al. Night-to-night sleep variability in older adults with and without chronic insomnia. Sleep Med. 2010;11(1):56-64. doi:10.1016/j.sleep.2009.02.010

35. Morin CM, Jarrin DC, Ivers H, Mérette C, LeBlanc M, Savard J. Incidence, persistence, and remission rates of insomnia over 5 years. JAMA Netw Open. 2020;3(11):e2018782. doi:10.1001/ jamanetworkopen.2020.18782

36. Janati Idrissi A, Lamkaddem A, Benouajjit A, et al. Sleep quality and mental health in the context of COVID-19 pandemic and lockdown in Morocco. Sleep Med. 2020;74(January):248-253. doi:10.1016/j. sleep.2020.07.045

37. Giuntella O, Hyde K, Saccardo S, Sadoff S. Lifestyle and mental health disruptions during COVID-19. Proc Natl Acad Sci USA. 2021;118(9):e2016632118. doi:10.1073/pnas.2016632118

38. Tähkämö L, Partonen T, Pesonen A-K. Systematic review of light exposure impact on human circadian rhythm. Chronobiol Int. 2019;36(2):151-170. doi:10.1080/07420528.2018.1527773

39. Touitou Y, Reinberg A, Touitou D. Association between light at night, melatonin secretion, sleep deprivation, and the internal clock: health impacts and mechanisms of circadian disruption. Life Sci. 2017;173:94-106. doi:10.1016/j.1fs.2017.02.008

40. Rezaei N, Grandner MA. Changes in sleep duration, timing, and variability during the COVID-19 pandemic: large-scale Fitbit data from 6 major US cities. Sleep Health. 2021;1-11. doi:10.1016/j. sleh.2021.02.008

41. Kantermann T, Juda M, Merrow M, Roenneberg T. The human circadian clock's seasonal adjustment is disrupted by daylight saving time. Curr Biol. 2007;17(22):1996-2000. doi:10.1016/j. cub.2007.10.025 


\section{Publish your work in this journal}

Nature and Science of Sleep is an international, peer-reviewed, open access journal covering all aspects of sleep science and sleep medicine, including the neurophysiology and functions of sleep, the genetics of sleep, sleep and society, biological rhythms, dreaming, sleep disorders and therapy, and strategies to optimize healthy sleep.
The manuscript management system is completely online and includes a very quick and fair peer-review system, which is all easy to use. Visit http://www.dovepress.com/testimonials.php to read real quotes from published authors. 